\title{
Multi-location Leakage Resilient Cryptography
}

\author{
Ali Juma ${ }^{1, \star}$, Yevgeniy Vahlis ${ }^{2}$, and Moti Yung ${ }^{3}$ \\ 1 Mozilla Corporation \\ ajuma@mozilla.com \\ 2 AT\&T Security Research Center \\ evahlis@att.com \\ 3 Google and Columbia University \\ my123@columbia.edu
}

\begin{abstract}
Understanding and modeling leakage in the context of cryptographic systems (connecting physical protection of keys and cryptographic operation) is an emerging area with many missing issues and hard to understand aspects. In this work we initiate the study of leakage out of cryptographic devices when the operation is inherently replicated in multiple locations. This setting (allowing the adversary access to leakage at different locations) arises naturally in cases like protocols, where different parties activate the same cryptographic function, or in the case of a global service providers (like cloud operators) which need to replicate the cryptographic function to allow for accessible and responsive services. We specifically deal with the theoretical setting of "leakage resilient cryptography," (modeling leakage as a bound associated with algorithmic steps), and in the most general model of continual leakage on memory, randomness (and thus computation) with periods of operation and refresh of private keys between them.

We first investigate public-key cryptography, and construct a multilocation leakage resilient signature scheme (with unbounded number of locations) with optimal (i.e., total $n(1-o(1))$ leakage) in a period, and $O(\log n)$ leakage during updates ( $n$ is the key size). The new crucial issue behind our scheme is how to maintain leakage at each location at the level of key leakage in the single location variant, even under parallel adaptive leakage at the different locations. We then construct a shared-symmetric-key authenticated session protocol that is resilient to leakage on both the sender and the receiver, and tolerates $O(\log n)$ bits of leakage per computation. We construct and utilize a single-location pseudorandom generator which is the first to tolerate continual leakage with only an efficient pseudorandom function as a primitive component. This protocol highlights the importance of protocol level "per message synchronization" against leakage adversaries. Interestingly, the construction is secure in spite of the entire randomness used in the refresh processes being publicly available.
\end{abstract}

\section{Introduction}

When a cryptographic function/ service is performed at more than one location, and an adversary attacks it, if the adversary has only black-box access to it

\footnotetext{
* This work was done while at the University of Toronto.
} 
and the scheme is stateless or state-synchronized (for correctness), then from security point of view, it does not seem to matter (i.e., and it does not require a new model) whether the scheme is operated from a single location or multiple ones (since the black box information revealed in a sequence of cryptographic application is insensitive to the location). However, when leakage is allowed to be part of the outputs, the adversary gets this added side-channel information 23 and as a result, may have different power, depending on whether the leakage is at a single location or if it comes from multiple locations.

The above observation is the motivation to this work, since the sensitivity to multiple location leakage is important to various systems settings, and we investigate this issue from the "leakage resistance cryptography" perspective. Note that multi location is natural when two parties in a protocol operate the same cryptographic service or when the same function is inserted in various devices (e.g., different cloud servers, different mobile devices within the same organization), etc.

The theme of this work is the design of secure cryptosystems under the existence of multiple locations. We consider both public key systems: in particular a signature scheme (while the methods we design may apply in more generality to encryption, etc.), and symmetric key systems: in particular session authentication protocols providing sender continual authentication to the receiver, based on shared pseudorandomness. We consider the model of continual leakage with no relaxation, i.e., where the leakage function is not only a result of computation but can be a function of the memory (state) and the randomness (i.e., the computation) as well. In these models, the parties need to go through periods of operation where leakage is given to the adversary and once the accumulated amount of leakage is large enough, the private keys are refreshed between periods (obviously if refresh is not possible, continued leakage may reveal over time the entire key bits, say one by one). We note that the above model is the strongest, compared with more limited types of leakage models that have been considered as well in the literature (such as: leakage in the presence of leakage-free components, leakage where "only computations leak," and only memory leakage (without leaking the randomness used in the cryptographic computation)).

\subsection{Multi-location Leakage Resilient Signature}

Since we consider continual leakage we have to make sure that we have a scheme where the total leakage in a period is only a fraction of the state (if the state is $l$ bits long we can allow at most $l(1-o(1))$ bits of information about the secret to be given to the adversary. Indeed, a few recent schemes have achieved such leakage in a period, allowing logarithmic leakage in the refresh process (e.g. [85]). In this version we concentrate on the signature scheme of [28].

In a multi-location setting, the scheme is replicated in various locations to allow better accessibility o the signing service, say, and necessarily these locations contain related key information (since the verification key is identical regardless of location). If the adversary collects enough information at different locations, each of which by itself is too small to break the key, the cumulative effect may 
nevertheless be that the adversary is in possession of enough bits to break the key (e.g., may collect all bits of a single replicated key). Thus, in the naive solution (which allows very limited leakage per location), we may restrict the amount of leakage at each location to be smaller than the total allowed per single location divided by the number of locations. This approach as we will show works sometimes, but sometimes fails!

We then turn into the challenging problem of constructing direct multilocation leakage resilient scheme which allows large leakges per location. Our starting point is a recent signature secure against continual leakage, where the crux behind this scheme is the fact that a key within a period is hidden within a large set of keys and the leakage within a period is simulatable by leakage correlated with a random value rather than the key [28. Then, the key is refreshed to another value within a large set of keys. A crucial point behind extending the signature to a multi location scheme which is refreshed at all locations periodically (when a bound on signatures at any single location is reached) is extension of the space from which private keys are drawn, so that multiple location leakage will also be simulatable by random values taking the two dimensions of variety of keys, namely, "periods" and "locations," into account when building the space of keys.

\subsection{Multi-location Symmetric-Key Authentication}

We next design a session authentication protocol from symmetric key whose goal is to continuously authenticate the sender to the receiver. A natural way of doing it is to base this on a stream cipher (i.e., a pseudorandom generator) which is run by both parties. Dziembowski and Pietrzak [12] and Pietrzak 31] gave leakage-resilient stream ciphers in the only-computation-leaks model (where the adversary gets a bounded size (logarithmic, in fact) leakage bits each time). Their seminal constructions use two pieces of memory connected by a public channel, and computation alternates between the two pieces. For an authenticated session we have two parties, a sender $A$ and a receiver $B$, where $A$ is sending message pieces to $B$, and we wish to ensure that an adversary cannot modify or reorder messages pieces, or insert message pieces of his own without this being detected by $B$. The adversary obtains leakage from both parties. The existing security definitions of leakage-resilient stream ciphers do not deal with this case at all. In fact, in the existing ciphers, an adversary that can cause parties $A$ and $B$ to "get out of sync" can attack the system and eventually learn the cipher's entire state. This suggests that we need a way to somewhat synchronize the stream cipher computations performed by the two parties.

Our construction, in turn, builds on Pietrzak's stream cipher construction 31, and uses a single piece of memory along with a source of strings that are chosen according to distribution of high min-entropy but are not kept secret (public min-entropy source), and are rather communicated between the parties. Our stream cipher uses a pseudo-random function generator $F_{s}:\{0,1\}^{n} \rightarrow\{0,1\}^{2 n}$. The initial secret state is randomly chosen $K_{0} \in\{0,1\}^{n}$. For each $i>0$, the $i$-th output is produced and the state is updated. $A$ authenticates the message using 
the output, while $B$ generates the next round high entropy string and sends it back for synchronization and update to $A$. Of course, the adversary controls the public channel and may insert strings of his choice (purporting to be sent by the other party) to induce a party to continue its computation; we show that such tampering by the adversary will be detected by $B$ when it attempts to verify the authenticity of the message pieces he receives; the construction allows continual leakage of logarithmic bits per round.

Remark: We note that Dodis et al [8] present a signature scheme which is multilocation leakage resilient (Theorem 7.6 in [8]), although they do not explicitly call it such. However, their scheme does not allow the adversary to obtain leakage on the randomness of signing. In contrast, in this work we define and present a signature scheme with multi-location resilience to full leakage (including signing randomness), and provide several generic theorems for obtaining multi-location leakage bounds for schemes that were intended to support only a single location.

\subsection{Related Work}

Side channel attacks 23] have often been shown to have devastating effects on the security of cryptographic schemes (some recent attacks that specifically pertain to general memory leakage are described in e.g., [1732 36, and others). As a result a significant effort has been wielded to design cryptographic schemes that provably withstand large classes of such attacks.

The influential theoretical works of Ishai, Sahai, and Wagner [19] and Micali and Reyzin 30] enable us to construct schemes under the "any computation, and only computation, leak information," model, which has led to many recent achievements. In contrast, memory leakage [1] (which, in some sense, can be traced to the original works of Shamir [34] and Rivest [33]) are produced as a function of the memory state itself. This type of leakage is orthogonal to computational leakage: an adversary can get memory leakage by probing memories even if the memories are not currently used in any computation (e.g., the coldboot attacks [17]). For example, the scheme of 98 is secure against memory attacks (even continual), but assumes that the signing process leaks no information. The most general model allows full leakage which includes leakage both from processing and memory.

The most demanding case for designing digital signature schemes seems to be the case of adaptive and continual full leakage that is available to the adversary from both computational and memory sources (without protection of sub-steps of computations). However, till recently there are no known schemes which achieve a digital signature scheme in this adversarial setting in the standard model. and without further relaxations. All known schemes with full (memory and processing) leakage either did not have a key update algorithm and thus are not continual (cf., [22]), have a key update algorithm but require some restrictions (e.g., 32, which requires an additional leakage-free master key), or are based on the random oracle model (with a relaxation of the definition of a "time period") [5]. Faust et al [13] construct signature schemes resilient to continual leakage in 
the only computation leaks model. Recently, two schemes have appeared with continual full leakage [284].

In the private key setting, Dziembowski and Pietrzak [12, and Pietrzak [31] describe the first stream ciphers resilient to continual leakage in the only computation leaks model. Our private key construction uses the works of [1231] as a starting point. Many other schemes dealt with the case of designing pseudorandom generation [12313911].

Faust et al [14] give a general compiler using secure hardware that protects an arbitrary circuit against continual leakage that can be modeled as a shallow $\left(A C^{0}\right)$ boolean circuit. Juma and Vahlis [20, and separately Goldwasser and Rothblum [16, give compilers that protect any algorithm against continual leakage (without complexity restrictions), using secure hardware. Recently, Dodis and Pietrzak [11] show how to build continual leakage resilient pseudorandom functions that are secure against non-adaptive leakage. Finally, Lewko et al 2625] show how to achieve leakage resilient Identity Based Encryption (IBE) and super-logarithmic leakage on key updates in signatures, and Chow et al [7] show an efficient leakage resilient IBE. A separate line of work studies strong leakage resilience in information theoretic implementation settings [35].

Finally, we mention a parallel rich line of work on tamper resistant cryptography [6 27|24 29|18 15]. Here, an adversary has the ability to modify, rather than observe, the state of the cryptographic primitive. Tamper resistant schemes provide security guarantees, even when the secret state is transformed through a tampering function adversarially chosen from a large class of functions. We remark that the techniques seem to be quite different from the ones employed to achieve leakage resilience. Indeed, studying the relation between tamper resistance and leakage resistance is an important direction.

Roadmap. In Section 2 we present our definitions of multi-location continuous leakage resilient signatures, and constructions. In Section 3 we present a shared key authenticated session protocol that is resilient to continuous leakage, and in Section 4 we present our variant of the Dziembowsky-Pietrzak leakage resilient stream cipher [12].

Notation. We write PPT to denote Probabilistic Polynomial Time. When we wish to fix the random bits of a PPT algorithm $M$ to a particular value, we write $M(x ; r)$ to denote running $M$ on input $x$ and randomness $r$. We write time $_{n}(M)$ to denote the running time of algorithm $M$ on security parameter $n$. We use $x \in_{\mathrm{R}} S$ to denote the fact that $x$ is sampled according to a distribution $S$. Similarly, when describing an algorithm we may write $x \leftarrow_{\mathrm{R}} S$ to denote the action of sampling an element from $S$ and storing it in a variable $x$. For a randomized algorithm $M$, we denote by $\operatorname{Rnd}[M]$ the space of its random coins. Namely, if $M$ uses at most $n_{M}$ random bits in any execution, then $\operatorname{Rnd}[M]=$ $\{0,1\}^{n_{M}}$. 


\section{Multi-location Leakage Resilience in the Public Key Setting}

In a multi-location setting, an adversary (or perhaps multiple colluding adversaries) simultaneously mount side channel attacks on multiple devices. When the devices contain unrelated data, and perform unrelated computations, such an attack should be viewed as a single side channel attack on each of the devices, since none of the other devices can provide any information that would help the attacker. The problem becomes much more serious when the devices contain correlated secret data. As an extreme case, consider a situation where multiple identical copies of a secret key are stored on several servers. Even if the cryptographic scheme where the key is used remained secure when the key is partially leaked, an adversary in a multi-location setting may be able to reconstruct the entire key from partial leakage from each of the locations. In this scenario, surely all reasonable security properties of the scheme can be broken. One possible approach to dealing with this apparent limitation is to restrict the total amount of leakage that the adversary can obtain across all copies of the key. Indeed, for some primitives (such as encryption) we show a straightforward reduction from multi-location leakage resilience with a bound on the total amount of leakage to single location leakage resilience. We note that, perhaps surprisingly, the same straightforward reduction fails for other primitives, such as signature schemes.

Note however that ideally, we want to obtain transformations where the leakage per location remains as large as the leakage bound in the single location setting. In Section 2.1 we give constructions of signature and encryption schemes where the leakage bound per location does not decrease with the number of different locations that maintain an equivalent copy of the key.

Overall, we note that extending multi-location leakage resilience to the continuous setting introduces several subtle challenges that do not appear when considering leakage from only a single location. Before describing these issues, we describe (informally) a generic transformation of a continuous leakage resilient signature scheme into a multi-location variant of itself. This will serve two purposes: firstly, it will help us illustrate the definitional issues that arise in multi-location continual leakage resilience. Secondly, our actual constructions and transformations can all be viewed as variants of the general approach that we describe here.

Consider a signature scheme that is resilient to continual leakage in the single location setting. As we have already discussed, such a scheme must have a key refresh procedure (otherwise, an adversary can eventually obtain the entire key). Moreover, suppose that the refresh procedure produces a new signing key chosen uniformly from the set of all valid keys that correspond to the public verification key that is generated once at the beginning. Now consider the following initialization procedure for an $n$-location signature scheme:

1. A public-private key pair $(v k, s k)$ is generated using the key generation procedure of the single location scheme. 
2. The key refresh procedure is used to produce $n$ random signing keys $s k_{1}, \ldots, s k_{n}$, all corresponding to the verification key $v k$.

3. Location $i$ receives key $s k_{i}$. Whenever location $i$ receives a request to update the key, it runs the refresh algorithm on its own key $s k_{i}$.

Essentially, each location maintains an independently chosen random signing key for $v k$, and when the leakage bound is reached for that specific location, the key is randomized. When $n=1$, this is exactly what happens in the single location setting (and thus for $n=1$ the security of the scheme trivially follows from its single location security). Consider now what happens when $n>1$ : at first glance it may seem that, because the keys at the different locations are independently chosen, leakage from one location would be completely useless in attacking another location. This turns out to be false for multiple reasons.

\subsection{Signature Schemes}

A signature scheme with key update $\mathcal{S G N}$ consists of four algorithms $\mathrm{Kg}$, Sig, Ver, and Update. The inputs and outputs of $\mathrm{Kg}$, Sig, and Ver are the same as in standard signature schemes. Update takes as input a secret key and a public key and outputs a new element of the secret key space. $\mathcal{S G \mathcal { N }}=(\mathrm{Kg}$, Sig, Ver, Update $)$ has to satisfy the following property:

- (Correctness) For any integers $n, m, i \geq 0$ and any message $M$, if we compute $\left(p k, s k_{1}^{(0)}, \ldots, s k_{m}^{(0)}\right) \leftarrow \operatorname{Gen}\left(1^{\kappa}, m\right), s k_{0} \leftarrow s k_{i}^{(0)}, s k_{1} \leftarrow \operatorname{Update}_{p k}\left(s k_{0}\right)$, $\ldots, s k_{n} \leftarrow$ Update $_{p k}\left(s k_{n-1}\right)$, and $\sigma \leftarrow \operatorname{Sig}\left(s k_{n}, M\right), \operatorname{Ver}(p k, M, \sigma)=1$ always holds.

We now define multi-location leakage resilience for signatures. Intuitively, the definition is a natural extension of the definitions of leakage resilient signatures that appeared in $28 / 5 / 8 / 410$. Intuitively, the adversary can submit signature queries and leakage queries that are directed at a specific location. For example, the adversary may submit a query that is interpreted as "Have the $i$ th signer sign message $m$, and obtain side-channel information $f\left(s k_{i}, r\right)$, where $r$ is the randomness used during signing, along with the resulting signature". The other types of queries are location specific signature queries without leakage (to allow longer periods between updates, as discussed in the introduction), and update queries. For update queries, we distinguish between synchronized updates where all locations refresh their keys simultaneously and unsynchronized updates where the adversary instructs the signer at some location $i$ to refresh his key. Finally, the adversary's goal is to produce a valid signature of a message that he has not submitted for signing in any of his queries.

\section{Experiment ExpMLSIG $\left(1^{n}, \mathcal{A}, \mathcal{S I G}\right)$ :}

Setup. The adversary submits an integer $m$, and the challenger runs $\operatorname{Gen}\left(1^{n}, m\right)$ to obtain a public verification key $p k$, and $m$ location secret keys $s k_{1}, \ldots, s k_{m}$.

Queries. $\mathcal{A}$ submits queries of the following three types: 
- Update queries.

Unsynchronized setting. Update queries of the form (update, $f, i$ ) where $f$ is a circuit satisfying $\left|f\left(s k_{i}, R\right)\right| \leq \rho_{U}\left(\left|s k_{i}\right|+|R|\right)$ for any $R$. If $L_{i}+\left|f\left(s k_{i}, R\right)\right| \leq$ $\rho_{M}\left|s k_{i}\right|$ holds, the challenger chooses $R \stackrel{\$}{\leftarrow}$ Rnd[Update] randomly, computes $s k_{i} \leftarrow$ Update $_{p k}\left(s k_{i}, R\right)$, sends $f\left(s k_{i}, R\right)$ back to $\mathcal{A}$, and sets $L_{i} \leftarrow\left|f\left(s k_{i}, R\right)\right|$. Otherwise, the challenger aborts.

Synchronized setting. Update queries of the form (update, $f_{1}, \ldots, f_{n}$ ) where $\left|f_{i}\left(s k_{i}, R\right)\right| \leq \rho_{U}\left(\left|s k_{i}\right|+|R|\right)$ for any $R$. If $L_{i}+\left|f\left(s k_{i}, R\right)\right| \leq \rho_{U}\left|s k_{i}\right|$ holds, the challenger chooses $R_{1}, \ldots, R_{n} \stackrel{\$}{\leftarrow}$ Rnd[Update] randomly, computes $s k_{i} \leftarrow$ Update $_{p k}\left(s k_{i} ; R_{i}\right)$, sends $\left(f_{i}\left(s k_{i}, R_{i}\right)\right)_{i=1}^{n}$ back to $\mathcal{A}$, and sets $L_{i} \leftarrow\left|f_{i}\left(s k_{i}, R_{i}\right)\right|$. Otherwise, the challenger aborts.

- Memory leak queries (leak, $f, i)$, where $f$ is a circuit. If $L_{i}+\left|f\left(s k_{i}\right)\right| \leq \rho_{M}\left|s k_{i}\right|$ holds, the challenger sends $f\left(s k_{i}\right)$ to adversary and resets $L_{i} \leftarrow L_{i}+\left|f\left(s k_{i}\right)\right|$. Otherwise, the challenger aborts.

- Signing queries (sig, $M, f, i)$ where $f$ is a circuit with $\left|f\left(s k_{i}, R\right)\right| \leq \rho_{S}\left(\left|s k_{i}\right|+\right.$ $|R|)$ for any $\left(s k_{i}, R\right)$. The challenger chooses $R \leftarrow \operatorname{Rnd}[\mathrm{Sig}]$ randomly, computes $\sigma \leftarrow \operatorname{Sig}\left(s k_{i}, M ; R\right)$ and sends $\left(\sigma, f\left(s k_{i}, R\right)\right)$ back to $\mathcal{A}$.

Challenge. Assuming the challenger did not abort, $\mathcal{A}$ outputs $\left(M_{*}, \sigma_{*}\right)$. It succeeds if $\operatorname{Ver}\left(p k, M_{*}, \sigma_{*}\right)=1$ holds and $\mathcal{A}$ never made query $\left(\operatorname{sig}, M_{*}, i\right)$ for any $i$.

Definition 1. Let $\rho_{G}, \rho_{U}, \rho_{M}$, and $\rho_{S}$ be elements of the real range $[0,1]$. We say that $\mathcal{S G \mathcal { N }}=($ Gen, Sig, Ver, Update $)$ is $\left(\rho_{G}, \rho_{U}, \rho_{M}, \rho_{S}\right)-E U-C M A-C M L$ secure (stand for existentially unforgeable under chosen message attack in the CML model) if no PPT adversary $\mathcal{A}$ succeeds in the experiment of ExpMLSIG with non-negligible probability. Here Rnd[Algo] denote the set of randomnesses for algorithm Algo.

In the full version of this paper [21] we show several negative results regarding generic transformations of single to multi location signature schemes, as well as a simple transformation that does work, under some restrictions on the base signature scheme. We now turn to a direct construction that achieves optimal leakage bounds.

Direct Multi-Location Leakage Resilience. The simple generic transformation (described in [21]) may not be satisfactory if the number of locations is very large. For instance, for a key of length 256 bits, even an optimally leakage resilient scheme that is transformed to a multi-location setting with more than 256 locations would be able to withstand less than one bit of leakage per location before the key has to be refreshed. This would require an extremely high refresh rate if even a small (but unknown) number of locations are suspected to leak information.

To address this, we turn to constructing signature and encryption schemes directly, that will withstand large amounts of leakage per location, and will allow the total amount of leakage among different locations to exceed the length of the key between updates. At the core of our constructions is a strengthening of the Leakage Resilient Subspaces Lemma from [5]. On a high level, the BKKV 
lemma can be described as follows: let $\mathcal{K}$ be an $n$-dimensional vector space, and let $Z_{1}, \ldots, Z_{l}, 1 \leq l<k$ be random elements in $\mathcal{K}$. Then, no adversary (even a computationally unbounded one) can distinguish between leakage from random samples from $\operatorname{Span}\left(Z_{1}, \ldots, Z_{l}\right)$ and random samples from $\mathcal{K}$. The key difference between the lemma in [5] and the one we present here is the ability of the adversary to leak on samples in parallel rather than sequentially: we show that even if the adversary breaks his leakage on a given sample into several rounds, where at each round he chooses the leakage function adaptively based on leakage from other samples, he is still unable to distinguish between random samples from $\mathcal{K}$ and from the $l$ dimensional subspace. We next describe the parallel leakage resilient subspace game:

Parallel-leakage resilient subspaces. Let $b \in\{0,1\}, n, \ell, m, \lambda$ be integers satisfying $n \geq \ell>m \geq 2, p$ be a prime, and $\mathcal{K}$ be a $n$-dimensional vector space over $\mathbb{Z}_{p}$. The following is the parallel leakage resilient subspace game, played with a computationally unbounded adversary $\mathcal{D}$ :

1. Let $Z_{1}, \ldots, Z_{\ell} \stackrel{\$}{\leftarrow} \mathcal{K}$. Initially a set $\Gamma=\left\{\Gamma_{1}, \ldots, \Gamma_{m}\right\}$ of size $m$ is sampled uniformly at random from $\mathcal{K}$ if $b=1$ and from $\operatorname{Span}\left(Z_{1}, \ldots, Z_{\ell}\right)$ if $b=0$.

2. The adversary can make leakage queries: (leak, $i, F$ ) where $i \in[\mathrm{m}]$ and $F$ : $\mathcal{K} \rightarrow\{0,1\}^{\lambda_{F}}, \mathcal{P}_{F} \subseteq \mathcal{P}$; and refresh queries: refresh. For a leakage query, the adversary is given $F\left(\Gamma_{i}\right)$, as long as $\sum_{F} \lambda_{F} \leq \lambda$ where the sum is over all the leakage functions $F$ that are applied to $\Gamma_{i}$ between two refresh queries. When the adversary submits a refresh query, $\left(\Gamma_{1}, \ldots, \Gamma_{m}\right)$ are assigned a random values from $\mathcal{K}$ if $b=1$ and random values from $\operatorname{Span}\left(Z_{1}, \ldots, Z_{\ell}\right)$ if $b=0$.

3. Finally, $\mathcal{D}$ is given $Z_{1}, \ldots, Z_{\ell}$, and it outputs a bit $b^{\prime}$.

We denote by $\operatorname{ExpLRS}(b, \mathcal{D})$ the above experiment with an adversary $\mathcal{D}$, and with the bit $b$ specified as a parameter. The output of $\operatorname{ExpRS}(b, \mathcal{D})$ is defined to be the output of $\mathcal{D}$ at the end of the experiment. We now state the central parallel leakage resilient subspaces lemma:

Lemma 1. Let $\mathcal{D}$ be an adversary for the above game. Then, for all $\delta \geq 0$, if $2^{\lambda_{\text {total }}} \leq p^{\ell-m-1} \delta^{2} / q^{2}$, then

$$
|\operatorname{Pr}[\operatorname{ExpLRS}(0, \mathcal{D})=0]-\operatorname{Pr}[\operatorname{ExpLRS}(1, \mathcal{D})=0]| \leq \delta .
$$

The proof of Lemma 1 appears in the full version of this paper [21].

\subsection{Construction}

We present a simple adaptation of the signature scheme of [28] to the multilocation setting. The modified construction allows us to achieve optimal leakage resilience, even when multiple versions of the key leak simultaneously. That is, the total amount of leakage across all locations between updates significantly exceeds the length of a single complete key (this is in contrast to the simple 
generic transformation, where the amount of leakage per location decreases as the number of locations increases to guarantee that the total amount does not exceed the size of a key). The changes required to the scheme and analysis are quite minimal. Indeed, the only substantial modification to the analysis is the use of the parallel leakage-resilient subspaces lemma (Lemma 1). For completeness, we describe the complete scheme here, and give a high level overview of the necessary modifications to the security analysis.

Our construction relies on the Symmetric External DDH assumption in bilinear groups (details of the assumption are given in [21]). The description of our scheme is as follows. Let $n \geq 3$ and $m$ be integers. Let Setup be a polytime algorithm that generates a group description $g k=(p, \mathbb{G}, \mathbb{H}, \mathbb{T}, \mathrm{e})$, as discussed above, where e : $\mathbb{G} \times \mathbb{H} \rightarrow \mathbb{T}$. For $\mathcal{H}=\left(\boldsymbol{H}_{0}, \boldsymbol{H}_{1}, \ldots, \boldsymbol{H}_{m}\right) \in\left(\mathbb{H}^{2}\right)^{m+1}$ and $M \in\{0,1\}^{m}$, we define a Water's hash function 38, $h$ as

$$
h_{g k}(\mathcal{H}, M)=\boldsymbol{H}_{0}+\sum_{k \in[m]} M_{k} \boldsymbol{H}_{k},
$$

where $M_{k}$ is the $k$-th bit of $M$. Let Prf and $\mathrm{Vrf}$ be the proof algorithm and the verification algorithm of the Groth-Sahai proof system (reviewed in [21]). Our signature scheme $\mathcal{S G \mathcal { N }}=(\mathrm{Kg}$, Update, Sig, Ver $)$ works as follows.

Key Generation Gen $\left(1^{\kappa}, m\right): g k \leftarrow(p, \mathbb{G}, \mathbb{H}, \mathbb{T}, \mathrm{e}) \leftarrow \operatorname{Setup}\left(1^{\kappa}\right), \boldsymbol{G} \leftarrow \mathbb{H}^{2}, \mathcal{H} \leftarrow$ $\left(\boldsymbol{H}_{0}, \boldsymbol{H}_{1}, \ldots, \boldsymbol{H}_{m}\right) \leftarrow\left(\mathbb{H}^{2}\right)^{m+1}$.

Randomly select $A \stackrel{\$}{\leftarrow} \mathbb{G}, Q \stackrel{\$}{\leftarrow} \mathbb{H}$, and $\boldsymbol{a}, \boldsymbol{q} \stackrel{\$}{\leftarrow} \mathbb{Z}_{p}^{n}$ satisfying $\langle\boldsymbol{a}, \boldsymbol{q}\rangle=0$ and compute $\boldsymbol{A} \leftarrow \boldsymbol{a} A, \boldsymbol{Q} \leftarrow \boldsymbol{q} Q$. Select $\boldsymbol{W}^{[0]} \stackrel{\$}{\leftarrow} \mathbb{H}^{n}$ randomly, compute $T \leftarrow \mathrm{e}\left(\boldsymbol{A}, \boldsymbol{W}^{[0]}\right)$. Then, the location specific keys are generated as: choose $s_{i} \stackrel{\$}{\leftarrow} \mathbb{Z}_{p}$, and set $\boldsymbol{W}_{i}^{[0]} \leftarrow \boldsymbol{W}^{[0]}+s_{i} \boldsymbol{Q}$. Outputs $p k \leftarrow(g k, \boldsymbol{G}, \mathcal{H}, \boldsymbol{A}, T, \boldsymbol{Q})$ and location specific private keys $\left(s k_{i}^{[0]}\right)_{i \in[m]}$.

Key Update Update Up $\left(s k^{[i]}\right)$ : Parse $p k$ and $s k^{[i]}$ as $(g k, \boldsymbol{G}, \mathcal{H}, \boldsymbol{A}, T, \boldsymbol{Q})$ and $\boldsymbol{W}^{[i]}$ respectively, select $s \stackrel{\$}{\leftarrow} \mathbb{Z}_{p}$ randomly, and output $s k^{[i+1]} \leftarrow \boldsymbol{W}^{[i+1]} \leftarrow$ $\boldsymbol{W}^{[i]}+s \boldsymbol{Q}$.

Signing $\operatorname{Sig}\left(s k^{[i]}, M\right)$ for $M \in\{0,1\}^{m}$ : Parse $p k$ and $s k^{[i]}$ as $(g k, \boldsymbol{G}, \mathcal{H}, \boldsymbol{A}, T, \boldsymbol{Q})$ and $\boldsymbol{W}^{[i]}$. Compute $\boldsymbol{H}_{M} \leftarrow h_{g k}(\mathcal{H}, M)$, set $\operatorname{crs}_{M} \leftarrow\left(\boldsymbol{G}, \boldsymbol{H}_{M}\right)$, and $\sigma \leftarrow$ $\operatorname{Prf}\left(g k, \operatorname{crs}_{M},(\boldsymbol{A}, T), \boldsymbol{W}^{[i]}\right)$ and output $\sigma$.

Verification $\operatorname{Ver}(p k, M, \sigma)$ : Parse $p k$ as $(g k, \boldsymbol{G}, \mathcal{H}, \boldsymbol{A}, T, \boldsymbol{Q})$, compute $\boldsymbol{H}_{M} \leftarrow$ $h_{g k}(\mathcal{H}, M)$, and set $\operatorname{crs}_{M} \leftarrow\left(\boldsymbol{G}, \boldsymbol{H}_{M}\right)$. If $\operatorname{Ver}\left(g k, c r s_{M},(\boldsymbol{A}, T), \sigma\right)=1$, output 1 . Otherwise, output 0.

Theorem 2. For any constants $c>0$ and any $\gamma=\Theta(1 / \sqrt{\kappa})$, the proposed scheme $\mathcal{S I G}$ is $\left(\rho_{G}, \rho_{U}, \rho_{M}, \rho_{S}\right)$-EU-CMA-CML secure under the $S X D H$ assumption. Here

$$
\left(\rho_{G}, \rho_{U}, \rho_{M}, \rho_{S}\right)=\left(\frac{c \cdot \log k}{n \log p}, \frac{c \cdot \log k}{n \log p}, 1-\frac{2+\gamma}{n}, 1-\frac{2+\gamma}{n}\right) .
$$

We can achieve the fraction $1-o(1)$ of leakage in signing and in memory by setting $n=\kappa$. 
Overview of the modifications to the analysis of the MTVY scheme. Essentially the analysis of the above scheme proceeds similarly to the analysis of the original (single-location) variant of 28. The main modification to the argument is to replace the use of the original leakage resilient subspace lemma from [5] with our parallel version, given by lemma 1] Specifically, in the proof of [28, Lemma 9] we replace the use of [28, Proposition 10] with our Lemma 11. Once we use Lemma 1, the subspace $\mathcal{W}$ remains information theoretically hidden from the adversary throughout the security, and therefore any successful forgery would yield a successful attack on the Independent Pre-Image Resistant Hash Function described in [28, Section 3]. We leave the full details of the analysis to the full version of this paper.

\section{Authenticated Session Protocols}

Next, we describe our definition and construction of a leakage resilient authenticated session protocol in the private key setting.

\subsection{Security Definition}

The intuitive goal of an authenticated session protocol involving two parties, the sender $A$ and the receiver $B$, where $A$ is sending message pieces $m_{1}, m_{2}, \ldots$, to $B$, is that $B$ can verify that the message pieces he receives are indeed those sent by $A$, in the same order. This should hold even when all message pieces $m_{i}$ sent by $A$ are adversarially chosen. Of course, the adversary has complete control of the public channel over which $A$ and $B$ are communicating. This means that he controls the timing and contents of all communication. In the leakage-resilient case, we strengthen the adversary by allowing him to obtain leakage on both parties. We are interested in the continual leakage setting, where the adversary obtains some bounded amount of leakage on each computation by each party but the total amount of leakage obtained by the adversary over the course of the execution of the protocol is unbounded. The leakage on each computation is computed by an adversarially-chosen function is applied to the inputs and randomness involved in the computation along with the entire state of the party performing the computation. This means that we do not rely on the only-computation-leaks assumption. In our case, we further strengthen the adversary by giving him all the entropy used by each party after the initial state. Equivalently, we require that $A$ and $B$ are deterministic but each have access to a (separate) source of public min-entropy; whenever a party obtains a string its source of high min-entropy strings, this string is also given to the adversary.

We begin by formally defining session protocols (we restrict our definition to protocols as ours with two flows per message, but the idea can be extended).

Definition 3. (Shared-private-key session protocol with public minentropy) $A$ shared-private-key session protocol with public min-entropy (which we will henceforth simply refer to as a session protocol) consists of deterministic 
polytime algorithms $\mathrm{EvalB}_{1}$ (producing message from B), EvalA (receiving the emssage from $B$ ), and $\mathrm{EvalB}_{2}$ (producing the message received from after evaluation), polynomials $s_{B}(n), \ell_{B}(n), s_{A}(n)$, and $\ell_{A}(n)$, and distribution ensembles $\left\{Z_{n}^{A}\right\}$ and $\left\{Z_{n}^{B}\right\}$ that satisfy the following properties for all $n \in \mathbb{N}$ :

1. $Z_{n}^{A}$ is a distribution over strings of length $s_{A}(n)$ such that $\mathbf{H}_{\infty}\left(Z_{n}^{A}\right) \geq$ $\log ^{2}(n)$. Similarly, $Z_{n}^{B}$ is a distribution over strings of length $s_{B}(n)$ such that $\mathbf{H}_{\infty}\left(Z_{n}^{B}\right) \geq \log ^{2}(n)$.

2. EvalB Eves $_{1}$ as input $K_{B} \in\{0,1\}^{n}$ and $r_{B} \in\{0,1\}^{s_{B}(n)}$, and outputs $\beta \in\{0,1\}^{\ell_{B}(n)}$ and $K_{B}^{\prime} \in\{0,1\}^{n}$ such that $\beta$ has prefix $r_{B}$.

Informally, the strings $K_{B}$ and $K_{B}^{\prime}$ are the state of party $B$ before and after it executes $\mathrm{EvalB}_{1}, r_{B}$ is the public min-entropy used by $\mathrm{EvalB}_{1}$, and $\beta$ is a flow from party $B$ to party $A$.

3. EvalA takes as input $K_{A} \in\{0,1\}^{n}, m \in\{0,1\}^{n}, \beta \in\{0,1\}^{\ell_{B}(n)}$, and $r_{A} \in\{0,1\}^{s_{A}(n)}$, and outputs $e \in\{0,1\}^{\ell_{A}(n)}$ and $K_{A}^{\prime} \in\{0,1\}^{n}$ such that $e$ has prefix $r_{A}$.

Informally, the strings $K_{A}$ and $K_{A}^{\prime}$ are the state of party $A$ before and after it executes EvalA, $m$ is a message piece that party $A$ would like to send to party $B, \beta$ is a flow from party $B$ to party $A, r_{A}$ is the public min-entropy used by EvalA, and $e$ is a flow from party $A$ to party $B$.

4. EvalB ${ }_{2}$ takes as input $K_{B} \in\{0,1\}^{n}, r_{B} \in\{0,1\}^{s_{B}(n)}$, and $e \in\{0,1\}^{\ell_{A}(n)}$, and outputs either $m \in\{0,1\}^{n}$ and $K_{B}^{\prime} \in\{0,1\}^{n}$ or a special message Fail.

Informally, the strings $K_{B}$ and $K_{B}^{\prime}$ are the state of party $B$ before and after it executes $\mathrm{EvalB}_{2}, r_{B}$ is the public min-entropy used by the immediately preceding run of $\mathrm{EvalB}_{1}$, $e$ is a flow from party $A$ to party $B$, and $m$ is a message piece received by party $B$.

5. For all $K \in\{0,1\}^{n}$, every polynomial $p(n)$, all $r_{A, 1}, r_{A, 2}, \ldots, r_{A, p(n)} \in$ $\{0,1\}^{s_{A}(n)}$, all $r_{B, 1}, r_{B, 2}, \ldots, r_{B, p(n)} \in\{0,1\}^{s_{B}(n)}$, and all sequences of message pieces $m_{1}, m_{2}, \ldots, m_{p(n)} \in\{0,1\}^{n}$, if we define $K_{A, 0}=K_{B, 0}=K$ and, for $1 \leq i \leq p(n)$, we iteratively define $K_{A, i}, K_{B, i}^{\prime}, K_{B, i}, e_{i}, \beta_{i}, m_{i}^{\prime}$ in the following manner:

$$
\begin{aligned}
\left(\beta_{i}, K_{B, i}^{\prime}\right) & \leftarrow \operatorname{EvalB}_{1}\left(K_{B, i-1}, r_{B, i}\right) \\
\left(e_{i}, K_{A, i}\right) & \leftarrow \operatorname{EvalA}\left(K_{A, i-1}, m_{i}, \beta_{i}, r_{A, i}\right) \\
\left(m_{i}^{\prime}, K_{B, i}\right) & \leftarrow \operatorname{EvalB}_{2}\left(K_{B, i}^{\prime}, r_{B, i}, e_{i}\right)
\end{aligned}
$$

then $m_{i}^{\prime}=m_{i}$ for all $1 \leq i \leq p(n)$.

Informally, this means that in the absence of an adversary, the message pieces output by party $B$ are exactly those sent by party $A$, in the same order.

We now define the security experiment for leakage-resilient authenticated session protocols. The adversary will be a family of polynomial-size circuits $C=\left\{C_{n}\right\}$. 
Letting $\lambda: \mathbb{N} \rightarrow \mathbb{N}$ be a function, we will say that an adversary $C$ is $\lambda(n)$ bounded if the leakage functions produced by $C_{n}$ over the course of the security experiment each have output length $\lambda(n)$. Fixing a session protocol

$$
\left(E v_{1} B_{1}, \text { EvalA, EvalB }{ }_{2}, s_{B}(n), \ell_{B}(n), s_{A}(n), \ell_{A}(n),\left\{Z_{n}^{A}\right\},\left\{Z_{n}^{B}\right\}\right)
$$

a function $\lambda: \mathbb{N} \rightarrow \mathbb{N}$, a $\lambda(n)$-bounded adversary $C=\left\{C_{n}\right\}$, and $n \in \mathbb{N}$, the security experiment proceeds as follows.

A string $K \in\{0,1\}^{n}$ is randomly chosen. We define $K_{A, 0}=K_{B, 0}=K$. Then, $C_{n}$ is allowed to run EvalA, EvalB ${ }_{1}$, and EvalB $\mathrm{B}_{2}$ in the following manner. $C_{n}$ may run these algorithms as many times as he wishes and in any order of his choice as long as for every $i>0$, the $(i+1)$-st invocation of EvalB ${ }_{1}$ does not

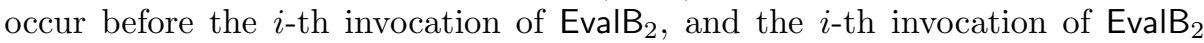
does not occur before the $i$-th invocation of EvalB ${ }_{1}$. (This restriction captures the fact that even though the adversary controls the public channel, party $B$ will still alternate between executing EvalB $B_{1}$ and executing EvalB $B_{2}$.) We now describe what happens when the adversary $C_{n}$ runs each algorithm.

- For $i>0$, the $i$-th invocation of EvalB 1 proceeds as follows. $C_{n}$ produces the description of a circuit $f_{B 1, i}:\{0,1\}^{n} \times\{0,1\}^{s_{B}(n)} \rightarrow\{0,1\}^{\lambda(n)}$. Then, $r_{B, i} \leftarrow Z_{n}^{B}$ is chosen. Next, $\left(\beta_{i}, K_{B, i}^{\prime}\right) \leftarrow \operatorname{EvalB}_{1}\left(K_{B, i-1}, r_{B, i}\right)$ and leak $k_{B 1, i} \leftarrow f_{B 1, i}\left(K_{B, i-1}, r_{B, i}\right)$ are computed. Finally, $C_{n}$ is given $\beta_{i}$ and $\operatorname{leak}_{B 1, i}$.

- For $i>0$, the $i$-th invocation of EvalA proceeds as follows. $C_{n}$ produces $m_{i} \in\{0,1\}^{n}$ and $\beta_{i}^{\prime} \in\{0,1\}^{\ell_{B}(n)}$, and the description of a circuit $f_{A, i}:\{0,1\}^{n} \times\{0,1\}^{s_{A}(n)} \rightarrow\{0,1\}^{\lambda(n)}$. Then, $r_{A, i} \leftarrow Z_{n}^{A}$ is randomly chosen. Next, $\left(e_{i}, K_{A, i}\right) \leftarrow \operatorname{EvalA}\left(K_{A, i-1}, m_{i}, \beta_{i}^{\prime}, r_{A, i}\right)$ and $l e a k_{A, i} \leftarrow$ $f_{A, i}\left(K_{A, i-1}, r_{A, i}\right)$ are computed 1 . Finally, $C_{n}$ is given $e_{i}$ and $l e a k_{A, i}$.

- For $i>0$, the $i$-th invocation of EvalB ${ }_{2}$ proceeds as follows. $C_{n}$ produces a string $e_{i}^{\prime} \in\{0,1\}^{\ell_{A}(n)}$ and the description of a circuit $f_{B 2, i}:\{0,1\}^{n} \rightarrow$ $\{0,1\}^{\lambda(n)}$. Then, $\left(m_{i}^{\prime}, K_{B, i}\right) \leftarrow \operatorname{EvalB}_{2}\left(K_{B, i}^{\prime}, r_{B, i}, e_{i}^{\prime}\right)$ and $l_{e a k_{B 2, i}} \leftarrow$ $f_{B 2, i}\left(K_{B, i}^{\prime}\right)$ are computed 2 ; if EvalB ${ }_{2}$ outputs Fail, the experiment ends immediately. If the $i$-th invocation of EvalA has previously occurred and $m_{i}^{\prime}=m_{i}, C_{n}$ is given leak $k_{B 2, i}$; otherwise, the experiment ends immediately.

Say that the final invocation of EvalB ${ }_{2}$ is the $j$-th invocation. Define $q_{C}(n)$ to be the probability that the $j$-th invocation of EvalB $\mathrm{B}_{2}$ does not output Fail and either EvalA has been invoked fewer than $j$ times or $m_{j}^{\prime} \neq m_{j}$.

Definition 4. (Leakage-resilient authenticated session protocol) Let $\lambda$ : $\mathbb{N} \rightarrow \mathbb{N}$ be a function. A session protocol is a $\lambda(n)$-leakage-resilient authenticated

\footnotetext{
${ }^{1}$ It is not necessary to provide $m_{i}$ or $\beta_{i}^{\prime}$ as inputs to $f_{A, i}$ since $C_{n}$ chose these values himself and hence he can simply hardcode them into $f_{A, i}$ if he wishes.

${ }^{2}$ It is not necessary to provide $r_{B, i}$ to $f_{B 2, i}$ since this was previously provided to $C_{n}$ as the prefix of $\beta_{i}$, and it is not necessary to provide $e_{i}^{\prime}$ to $f_{B 2, i}$ since $C_{n}$ chose this value himself.
} 
session protocol if for every $\lambda(n)$-bounded adversary $C$ as above, we have $q_{C}(n) \leq$ $1 / n^{d}$ for all $d$ and sufficiently large $n$.

\subsection{Our Construction}

In our construction, only party $B$ requires a source of public min-entropy. Accordingly, to simplify notation, we use $Z_{n}$ rather that $Z_{n}^{B}$ to denote the high min-entropy distribution used by $B$.

Given pseudo-random function generators $F:\{0,1\}^{n} \times\{0,1\}^{n} \rightarrow\{0,1\}^{2 n}$ and $F^{\prime}:\{0,1\}^{n} \times\{0,1\}^{n} \rightarrow\{0,1\}^{n}$, and given a distribution ensemble $\left\{Z_{n}\right\}$ such that for all $n, Z_{n}$ is a distribution over $\{0,1\}^{n}$ and $\mathbf{H}_{\infty}\left(Z_{n}\right) \geq \log ^{2}(n)$, we construct a leakage-resilient authenticated session protocol SP as follows.

EvalB ${ }_{1}$ : On input $\left(K_{B}, r_{B}\right)$, where $K_{B} \in\{0,1\}^{n}$ and $r_{B} \in\{0,1\}^{n}$, EvalB 1 lets $K_{B}^{\prime}=K_{B}$ and $\beta=r_{B}$, and outputs $\left(\beta, K_{B}^{\prime}\right)$.

EvalA: On input $\left(K_{A}, m, \beta\right)$, where $K_{A}, m \in\{0,1\}^{n}$ and $\beta \in\{0,1\}^{n}$, EvalA computes $K_{A}^{\prime}|| X_{A} \leftarrow F_{K_{A}}(\beta)$ (where $\left|K_{A}^{\prime}\right|=\left|X_{A}\right|=n$ ) and $\alpha=F_{X_{A}}^{\prime}(m)$, lets $e=\langle m, \alpha\rangle$, and outputs $\left(e, K_{A}^{\prime}\right)$.

EvalB ${ }_{2}$ : On input $\left(K_{B}, r_{B}, e^{\prime}\right)$, where $K_{B} \in\{0,1\}^{n}, r_{B} \in\{0,1\}^{n}$, and $e^{\prime} \in$ $\{0,1\}^{2 n}$, EvalB 2 parses $\left\langle m^{\prime}, \alpha^{\prime}\right\rangle \leftarrow e^{\prime}$, computes $K_{B}^{\prime} \| X_{B} \leftarrow F_{K_{B}}\left(r_{B}\right)$ (where $\left.\left|K_{B}^{\prime}\right|=\left|X_{B}\right|=n\right)$, and $\alpha=F_{X_{B}}^{\prime}\left(m^{\prime}\right)$. If $\alpha^{\prime}=\alpha$, EvalB ${ }_{2}$ outputs $\left(m^{\prime}, K_{B}^{\prime}\right)$; otherwise, EvalB ${ }_{2}$ outputs Fail.

It is not hard to see that SP satisfies the definition of a session protocol. The idea is that parties $A$ and $B$ both run a stream cipher (see Section 4) starting from the same key and using the same inputs, and use the $i$-th output $X_{i}$ to compute a signature $F_{X_{i}}^{\prime}\left(m_{i}\right)$ of the $i$-th message piece $m_{i}$.

Theorem 5. For all $c>0, \mathrm{SP}$ is a $c \log n$-leakage-resilient authenticated session protcol.

For the details of the proof of Theorem 5 we direct the reader to [21].

\section{Stream Cipher Construction}

In this section, we present our modified version of Pietrzak's stream cipher. The main purpose of our construction is to prove Theorem 6. which in turn is used in the proof of Theorem 5. Our construction uses only a single piece of memory but requires a public source of min-entropy. We believe that the construction below and its analysis are of independent interest due to the involved analysis of the leakage resilient stream cipher with public randomness.

The construction. Let $F:\{0,1\}^{n} \times\{0,1\}^{n} \rightarrow\{0,1\}^{2 n}$ be a pseudo-random function. Let $\left\{Z_{n}\right\}$ be such that for all $n, Z_{n}$ is a distribution over strings of length $n$ and $\mathbf{H}_{\infty}\left(Z_{n}\right) \geq \log ^{2}(n)$. The initial state is $K_{0}$, where $K_{0} \in\{0,1\}^{n}$ is randomly chosen. For each $i>0$, the $i$-th round consists of: 
1. $R_{i} \leftarrow Z_{n}$ is chosen.

2. $K_{i} \| X_{i} \leftarrow F_{K_{i-1}}\left(R_{i}\right)$.

3. The new state is $K_{i}$.

The adversary's interaction. Fix $c>0 . \mathrm{A}(c \log n)$-bounded adversary interacts as follows. For each $i>0$ :

1. Before round $i$, the adversary outputs the description of a function $f_{i}$ : $\{0,1\}^{2 n} \rightarrow\{0,1\}^{c \log n}$.

2. After round $i$, the adversary sees $R_{i}, X_{i}, f_{i}\left(K_{i-1}, R_{i}\right)$.

\subsection{Security Analysis}

We begin by defining some notation.

For an adversary $A$, we will use $\mathrm{real}_{i}$ to denote the adversary's view after the first $i$ rounds along with the corresponding $X_{j}$. That is,

$$
\operatorname{real}_{i}=\left\langle R_{1}, f_{1}\left(K_{0}, R_{1}\right), X_{1}, R_{2}, f_{2}\left(K_{1}, R_{2}\right), X_{2}, \ldots, R_{i}, f_{i}\left(K_{i-1}, R_{i}\right), X_{i}\right\rangle
$$

Note that the $f_{j}$ are not fixed functions, but rather are chosen adaptively by the adversary $A$ as described in Section 4 .

We will also define a version of $\mathrm{real}_{i}$ that includes an additional round where there is no leakage. Specifically, we define

$$
\operatorname{real}_{i}^{+}=\left\langle\operatorname{real}_{i}, R_{i+1}, K_{i+1}, X_{i+1}\right\rangle
$$

That is, $\mathrm{real}_{i}^{+}$includes the inputs and outputs of an additional leak-free round along with the entire state at the end of that round.

Theorem 6. Let $F:\{0,1\}^{n} \times\{0,1\}^{n} \rightarrow\{0,1\}^{2 n}$ be a pseudo-random function. Let $K^{\prime}$ and $X^{\prime}$ be independent random variables that are each uniformly distributed over $\{0,1\}^{n}$. Let $\left\{Z_{n}\right\}$ be such that for all $n, Z_{n}$ is a distribution over strings of length $n$ and $\mathbf{H}_{\infty}\left(Z_{n}\right) \geq \log ^{2}(n)$. For all $c>0, d>0, e>0$, every function $p: \mathbb{N} \rightarrow \mathbb{N}$, sufficiently large $n$, all $(c \log n)$-bounded adversaries $A$ interacting as described in section 4 and obtaining leakage for $p(n)$ rounds, and all adversaries $D$ such that $2 \cdot \operatorname{size}(A)+\operatorname{size}(D)+p(n) \operatorname{size}(F) \leq n^{e}$,

$$
\left|\operatorname{Pr}\left[D\left(\operatorname{real}_{p(n)}^{+}\right)=1\right]-\operatorname{Pr}\left[D\left(\operatorname{real}_{p(n)}, R_{p(n)+1}, K^{\prime}, X^{\prime}\right)=1\right]\right| \leq \frac{6 p(n)+6}{n^{d}} .
$$

Specifically, for sufficiently large $n$ (depending only on $c$, $d$, and $e$ ), if there exists an adversary $D$ breaking the above, then there exists an adversary of size $n^{e+8 d+2 c+8}$ breaking $F$ with advantage $1 / n^{5 d+2 c+3}$.

The details of the proof of Theorem 6] are given in [21. The high-level approach is similar to that of Pietrzak [31], but there are differences in the details, due to the differences in our security models. 


\section{References}

1. Akavia, A., Goldwasser, S., Vaikuntanathan, V.: Simultaneous Hardcore Bits and Cryptography Against Memory Attacks. In: Reingold, O. (ed.) TCC 2009. LNCS, vol. 5444, pp. 474-495. Springer, Heidelberg (2009)

2. Alwen, J., Dodis, Y., Naor, M., Segev, G., Walfish, S., Wichs, D.: Public-Key Encryption in the Bounded-Retrieval Model. In: Gilbert, H. (ed.) EUROCRYPT 2010. LNCS, vol. 6110, pp. 113-134. Springer, Heidelberg (2010)

3. Alwen, J., Dodis, Y., Wichs, D.: Leakage-Resilient Public-Key Cryptography in the Bounded-Retrieval Model. In: Halevi, S. (ed.) CRYPTO 2009. LNCS, vol. 5677, pp. 36-54. Springer, Heidelberg (2009)

4. Boyle, E., Segev, G., Wichs, D.: Fully Leakage-Resilient Signatures. In: Paterson, K.G. (ed.) EUROCRYPT 2011. LNCS, vol. 6632, pp. 89-108. Springer, Heidelberg (2011)

5. Brakerski, Z., Kalai, Y.T., Katz, J., Vaikuntanathan, V.: Overcoming the hole in the bucket: Public-key cryptography resilient to continual memory leakage. In: 51st Annual Symposium on Foundations of Computer Science, pp. 501-510. IEEE Computer Society Press (2010)

6. Choi, S.G., Kiayias, A., Malkin, T.: BiTR: Built-in Tamper Resilience. In: Lee, D.H., Wang, X. (eds.) ASIACRYPT 2011. LNCS, vol. 7073, pp. 740-758. Springer, Heidelberg (2011), Cryptology ePrint Archive, Report 2010/503 (2010), http://eprint.iacr.org/

7. Chow, S.S.M., Dodis, Y., Rouselakis, Y., Waters, B.: Practical leakage-resilient identity-based encryption from simple assumptions. In: Al-Shaer, E., Keromytis, A.D., Shmatikov, V. (eds.) ACM CCS 2010: 17th Conference on Computer and Communications Security, pp. 152-161. ACM Press (October 2010)

8. Dodis, Y., Haralambiev, K., López-Alt, A., Wichs, D.: Cryptography against continuous memory attacks. In: 51st Annual Symposium on Foundations of Computer Science, pp. 511-520. IEEE Computer Society Press (2010)

9. Dodis, Y., Haralambiev, K., López-Alt, A., Wichs, D.: Efficient Public-Key Cryptography in the Presence of Key Leakage. In: Abe, M. (ed.) ASIACRYPT 2010. LNCS, vol. 6477, pp. 613-631. Springer, Heidelberg (2010)

10. Dodis, Y., Lewko, A., Waters, B., Wichs, D.: Storing secrets on continually leaky devices. Cryptology ePrint Archive, Report 2011/369 (2011), http://eprint.iacr.org/; To appear in FOCS 2011

11. Dodis, Y., Pietrzak, K.: Leakage-Resilient Pseudorandom Functions and SideChannel Attacks on Feistel Networks. In: Rabin, T. (ed.) CRYPTO 2010. LNCS, vol. 6223, pp. 21-40. Springer, Heidelberg (2010)

12. Dziembowski, S., Pietrzak, K.: Leakage-resilient cryptography. In: 49th Annual Symposium on Foundations of Computer Science, pp. 293-302. IEEE Computer Society Press (October 2008)

13. Faust, S., Kiltz, E., Pietrzak, K., Rothblum, G.N.: Leakage-Resilient Signatures. In: Micciancio, D. (ed.) TCC 2010. LNCS, vol. 5978, pp. 343-360. Springer, Heidelberg (2010)

14. Faust, S., Rabin, T., Reyzin, L., Tromer, E., Vaikuntanathan, V.: Protecting Circuits from Leakage: the Computationally-Bounded and Noisy Cases. In: Gilbert, H. (ed.) EUROCRYPT 2010. LNCS, vol. 6110, pp. 135-156. Springer, Heidelberg (2010) 
15. Gennaro, R., Lysyanskaya, A., Malkin, T., Micali, S., Rabin, T.: Algorithmic Tamper-Proof (ATP) Security: Theoretical Foundations for Security against Hardware Tampering. In: Naor, M. (ed.) TCC 2004. LNCS, vol. 2951, pp. 258-277. Springer, Heidelberg (2004)

16. Goldwasser, S., Rothblum, G.N.: Securing Computation against Continuous Leakage. In: Rabin, T. (ed.) CRYPTO 2010. LNCS, vol. 6223, pp. 59-79. Springer, Heidelberg (2010)

17. J. Alex Halderman, Schoen, S.D., Heninger, N., Clarkson, W., Paul, W., Calandrino, J.A., Feldman, A.J., Appelbaum, J., Felten, E.W.: Lest we remember: Cold boot attacks on encryption keys. In: van Oorschot, P.C. (ed.) USENIX Security Symposium, pp. 45-60. USENIX Association (2008)

18. Ishai, Y., Prabhakaran, M., Sahai, A., Wagner, D.: Private Circuits II: Keeping Secrets in Tamperable Circuits. In: Vaudenay, S. (ed.) EUROCRYPT 2006. LNCS, vol. 4004, pp. 308-327. Springer, Heidelberg (2006)

19. Ishai, Y., Sahai, A., Wagner, D.: Private Circuits: Securing Hardware against Probing Attacks. In: Boneh, D. (ed.) CRYPTO 2003. LNCS, vol. 2729, pp. 463-481. Springer, Heidelberg (2003)

20. Juma, A., Vahlis, Y.: Protecting Cryptographic Keys against Continual Leakage. In: Rabin, T. (ed.) CRYPTO 2010. LNCS, vol. 6223, pp. 41-58. Springer, Heidelberg (2010)

21. Juma, A., Vahlis, Y., Yung, M.: Multi-Location Leakage Resilient Cryptography. In: Fischlin, M., Buchmann, J., Manulis, M. (eds.) PKC 2012. LNCS, vol. 7293, pp. 504-521. Springer, Heidelberg (2012), http://eprint.iacr.org/

22. Katz, J., Vaikuntanathan, V.: Signature Schemes with Bounded Leakage Resilience. In: Matsui, M. (ed.) ASIACRYPT 2009. LNCS, vol. 5912, pp. 703-720. Springer, Heidelberg (2009)

23. Kocher, P.C.: Timing Attacks on Implementations of Diffie-Hellman, RSA, DSS, and Other Systems. In: Koblitz, N. (ed.) CRYPTO 1996. LNCS, vol. 1109, pp. 104-113. Springer, Heidelberg (1996)

24. Komano, Y., Ohta, K., Miyake, H., Shimbo, A.: Algorithmic Tamper Proof (ATP) Counter Units for Authentication Devices Using PIN. In: Abdalla, M., Pointcheval, D., Fouque, P.-A., Vergnaud, D. (eds.) ACNS 2009. LNCS, vol. 5536, pp. 306-323. Springer, Heidelberg (2009)

25. Lewko, A., Lewko, M., Waters, B.: How to leak on key updates. Cryptology ePrint Archive, Report 2010/562 (2010), http://eprint.iacr.org/

26. Lewko, A., Rouselakis, Y., Waters, B.: Achieving Leakage Resilience through Dual System Encryption. In: Ishai, Y. (ed.) TCC 2011. LNCS, vol. 6597, pp. 70-88. Springer, Heidelberg (2011)

27. Liu, F.-H., Lysyanskaya, A.: Algorithmic Tamper-Proof Security under Probing Attacks. In: Garay, J.A., De Prisco, R. (eds.) SCN 2010. LNCS, vol. 6280, pp. 106-120. Springer, Heidelberg (2010)

28. Malkin, T., Teranishi, I., Vahlis, Y., Yung, M.: Signatures Resilient to Continual Leakage on Memory and Computation. In: Ishai, Y. (ed.) TCC 2011. LNCS, vol. 6597, pp. 89-106. Springer, Heidelberg (2011)

29. Mateus, P., Vaudenay, S.: On Tamper-Resistance from a Theoretical Viewpoint. In: Clavier, C., Gaj, K. (eds.) CHES 2009. LNCS, vol. 5747, pp. 411-428. Springer, Heidelberg (2009)

30. Micali, S., Reyzin, L.: Physically Observable Cryptography (Extented Abstract). In: Naor, M. (ed.) TCC 2004. LNCS, vol. 2951, pp. 278-296. Springer, Heidelberg (2004) 
31. Pietrzak, K.: A Leakage-Resilient Mode of Operation. In: Joux, A. (ed.) EUROCRYPT 2009. LNCS, vol. 5479, pp. 462-482. Springer, Heidelberg (2009)

32. Ristenpart, T., Tromer, E., Shacham, H., Savage, S.: Hey, you, get off of my cloud: exploring information leakage in third-party compute clouds. In: Al-Shaer, E., Jha, S., Keromytis, A.D. (eds.) ACM CCS 2009: 16th Conference on Computer and Communications Security, pp. 199-212. ACM Press (November 2009)

33. Rivest, R.L.: All-or-Nothing Encryption and the Package Transform. In: Biham, E. (ed.) FSE 1997. LNCS, vol. 1267, pp. 210-218. Springer, Heidelberg (1997)

34. Shamir, A.: How to share a secret. Communications of the Association for Computing Machinery 22(11), 612-613 (1979)

35. Standaert, F.-X., Malkin, T.G., Yung, M.: A Unified Framework for the Analysis of Side-Channel Key Recovery Attacks. In: Joux, A. (ed.) EUROCRYPT 2009. LNCS, vol. 5479, pp. 443-461. Springer, Heidelberg (2009)

36. Tromer, E., Osvik, D.A., Shamir, A.: Efficient cache attacks on AES, and countermeasures. Journal of Cryptology 23(1), 62-74 (2010)

37. Veyrat-Charvillon, N., Standaert, F.-X.: Generic Side-Channel Distinguishers: Improvements and Limitations. In: Rogaway, P. (ed.) CRYPTO 2011. LNCS, vol. 6841, pp. 354-372. Springer, Heidelberg (2011)

38. Waters, B.: Efficient Identity-Based Encryption Without Random Oracles. In: Cramer, R. (ed.) EUROCRYPT 2005. LNCS, vol. 3494, pp. 114-127. Springer, Heidelberg (2005)

39. Yu, Y., Standaert, F.-X., Pereira, O., Yung, M.: Practical leakage-resilient pseudorandom generators. In: Al-Shaer, E., Keromytis, A.D., Shmatikov, V. (eds.) ACM CCS 2010: 17th Conference on Computer and Communications Security, pp. 141151. ACM Press (October 2010) 